\title{
Regulatory functions of NK cells during infections and cancer
}

\author{
Norberto W. Zwirner ${ }^{1,2}$ (iD $\quad$ Carolina I. Domaica ${ }^{1}$ (iD | Mercedes B. Fuertes $^{1}$ (iD
}

\author{
${ }^{1}$ Laboratorio de Fisiopatología de la Inmunidad \\ Innata, Instituto de Biología y Medicina \\ Experimental (IBYME), Consejo Nacional de \\ Investigaciones Científicas y Técnicas \\ (CONICET), Buenos Aires, Argentina \\ ${ }^{2}$ Departamento de Química Biológica, Facultad \\ de Ciencias Exactas y Naturales, Universidad de \\ Buenos Aires, Buenos Aires, Argentina \\ Correspondence \\ Mercedes B. Fuertes, IBYME-CONICET, Vuelta \\ de Obligado 2490, Buenos Aires, Argentina. \\ Email:merfuer@gmail.com
}

\begin{abstract}
After recognition, NK cells can kill susceptible target cells through perforin-dependent mechanisms or by inducing death receptor-mediated apoptosis, and they can also secrete cytokines that are pivotal for immunomodulation. Despite the critical role as effector cells against tumors and virus-infected cells, NK cells have been implicated in the regulation of T cell-mediated responses in different models of autoimmunity, transplantation, and viral infections. Here, we review the mechanisms described for NK cell-mediated inhibition of adaptive immune responses, with spotlight on the emerging evidence of their regulatory role that shapes antitumor immune responses.
\end{abstract}

KEYWORDS

NK cell, immunoregulation, tumor

\section{1 | INTRODUCTION}

NK cells constitute the third most abundant lymphoid cell population in blood of humans. Evidence obtained from mouse studies and the analysis of patients with rare primary immunodeficiencies characterized by the absence of NK cells or the presence of functionally deficient NK cells unraveled their critical role during immunity against viral infections. ${ }^{1-3}$ Experimental evidence obtained in mice also positioned them as major players in antitumor immunity. ${ }^{4,5}$ In humans, such role was inferred from a large longitudinal study where an association between "natural cytotoxicity" in blood and susceptibility to develop different malignancies was observed. ${ }^{6}$ NK cells also exert immunoregulatory effects in different physiologic and pathologic conditions, skewing the adaptive immune response toward a Th1and cytotoxic CD8 T cell-mediated immune response. ${ }^{7-9}$ In addition, it has been established that NK cells are relatively abundant in different tissues, contributing to the local immunosurveillance against pathogens. ${ }^{10,11}$

The discovery of a new family of innate immune cells named innate lymphoid cells (ILCS) that play an essential role in the initiation, regulation, and resolution of inflammation has led to a reclassification of NK cells as one of the components of this extended family of innate

Abbreviations: DCs, dendritic cells; EAE, experimental autoimmune encephalomyelitis; EAU, experimental autoimmune uveitis; $\mathrm{HBV}$, hepatitis $B$ virus; $\mathrm{HCV}$, hepatitis $\mathrm{C}$ virus; ILCs, innate lymphoid cells; LCMV, lymphocytic choriomeningitis virus; MCMV, murine cytomegalovirus; NSCLC, non-small cell lung cancer; PD-L1, programmed death-ligand 1; Tfh, follicular helper CD4 T cells; TIL, tumor-Infiltrating Lymphocytes; Treg, regulatory T cell. immune cells of lymphoid lineage. ${ }^{12}$ All ILCs lack an antigenic receptor and, with the exception of NK cells, are sparse in lymphoid organs, but enriched in mucosa and skin. ${ }^{13}$

Although classically it was assumed that NK cells remain usually in a nonactivated status or silenced due to the expression of a vast array of inhibitory receptors, such as KIRs, NKG2A, 2B4, TIGIT, and others, recent evidence obtained by single-cell transcriptome analysis demonstrated that different human NK cell subpopulations from bone marrow and blood express genes that indicate the occurrence of a homeostatic activation. ${ }^{14}$ Nonetheless, when NK cells encounter tumor or infected host cells that display a dysregulated expression of ligands for activation receptors such as NKG2D, NKp46, and NKp30, ${ }^{5}$ NK cells usually become activated and trigger a cytotoxic response against these susceptible target cells and also begin to secrete cytokines (in particular IFN- $\gamma$ ) and chemokines. ${ }^{10,15}$ NK cells can kill virus-infected or tumor cells through the release of lytic granules containing granzyme and perforin or by inducing death receptor-mediated apoptosis via the expression of FasL or TRAIL. ${ }^{16}$ Moreover, NK cells become activated by diverse proinflammatory cytokines, in particular IL-12, IL-15, and IL-18. ${ }^{17}$

From a functional perspective, mouse and human NK cells are quite similar (both are cytotoxic and produce proinflammatory cytokines). However, they differ in some phenotypic features. In humans, NK cells are defined by the absence of expression of CD3 and by the expression of CD56 and CD16 (the FcR $\gamma$ Illa). According to the expression of CD56 and CD16, they are further subdivided into 2 major subpopulations. ${ }^{18}$ Nearly $90 \%$ of NK cells in 
peripheral blood exhibit a $\mathrm{CD} 3^{-} \mathrm{CD} 56^{\mathrm{dim}} \mathrm{CD} 16^{+}$phenotype, and exhibit a high content of perforin and granzymes (and consequently, display a strong cytotoxic activity); the rest of NK cells in blood display a $\mathrm{CD}^{-}{ }^{-} \mathrm{CD} 56^{\text {bright }} \mathrm{CD} 16^{\mathrm{dim} /-}$ phenotype, express CD25, and mostly produce immunoregulatory cytokines in response to different stimuli. ${ }^{18-22}$ These cells prevail in secondary lymph nodes and, through the secretion of cytokines, instruct dendritic cells (DCs) to generate Th1- and cytotoxic CD8 T cell-skewed responses, shaping in this way the adaptive immunity. ${ }^{23,24}$ In vitro evidence and studies from certain primary immunodeficiencies indicate that CD56 ${ }^{\text {bright }} \mathrm{CD} 16^{\mathrm{dim} /-}$ cells constitute a precursor of the most mature $\mathrm{CD} 56^{\mathrm{dim}} \mathrm{CD} 16^{+}$ cells. ${ }^{25-29}$ However, in mice and because they do not express CD56, NK cells are characterized as CD3-CD49b cells, but in some particular strains such as $\mathrm{C} 57 \mathrm{BL} / 6$, they can also be characterized as $\mathrm{CD}^{-} \mathrm{CD} 161 \mathrm{~b} / \mathrm{CD} 161 \mathrm{c}^{+}$cells (CD3 ${ }^{-} \mathrm{NK} 1.1^{+}$cells). ${ }^{30}$ In addition, 4 subpopulations of mouse NK cells are currently recognized, based on the expression of CD27 and CD11b, with phenotypes CD27- ${ }^{-} D 11 b^{-}$, $\mathrm{CD} 27^{+} \mathrm{CD} 11 b^{-}, \mathrm{CD} 27^{+} \mathrm{CD} 11 b^{+}$, and $\mathrm{CD} 27^{--} \mathrm{CD} 11 b^{+}$from the most immature to the most mature NK cell subpopulations. ${ }^{31,32}$

Although an array of diverse surface receptors confers NK cells the ability to sense ligands expressed on target cells and respond accordingly, NK cell also senses proinflammatory and immunosuppressive cytokines produced by different cells of myeloid origin. ${ }^{33}$ Moreover, NK cells also express TLR and respond to the presence of their specific ligands. ${ }^{34,35}$ In addition, cooperation between cytokines for NK cell activation and development of effector function has been described. ${ }^{9,36,37}$ Therefore, NK cells can integrate multiple signals from their environment and adjust their effector functions to optimize the extent of their response to pathogens and tumor cells.

Despite their critical role as effectors against tumors and pathogens, more recently NK cells have been implicated in the negative regulation of adaptive immune responses. In this review, we discuss the role of NK cells regulating adaptive immunity both in physiologic and in pathologic conditions, with their regulatory functions during antitumor immunity on the spotlight.

\section{2 | REGULATORY FUNCTIONS OF NK CELLS DURING INFECTIONS}

During infections, strong adaptive immune responses need to be tightly controlled to optimize efficient pathogen elimination while protecting the host from immunopathology. Accumulating evidence highlights the role of NK cells as regulators of adaptive immune responses. Several reports show evidence of the ability of NK cells to directly eliminate activated T cells in a perforin-dependent manner. ${ }^{38,39}$ Early studies demonstrated that mouse CD4 and CD8 T cells that have been activated by antigen presenting cells ${ }^{38}$ and human CD4 and CD8 T cells activated after superantigen, alloantigen, or specific antigenic peptide-stimulation, ${ }^{39}$ up-regulate the expression of NKG2D ligands, which makes them susceptible to recognition and elimination by IL-2-activated NK cells in a NKG2D- and perforin- dependent manner in vitro. ${ }^{38,39}$ Moreover, the regulatory role of NK cells was also appreciated in vivo in different physiopatholog situations, as detailed in the next sections.

\section{1 | Regulatory functions of NK cells during viral infections}

Studies using lymphocytic choriomeningitis virus (LCMV) infection unraveled that NK cells can negatively regulate the magnitude of the antiviral response. LCMV constitutes an excellent experimental model that has been widely used to address tissue-specific tolerance and its underlying mechanisms. ${ }^{40,41}$ Although LCMV infection strongly activates NK cells, virus elimination is NK cell-independent. ${ }^{42}$ Besides, in 2B4-deficient LCMV-infected mice, NK cells were able to eliminate activated CD8 $\mathrm{T}$ cells in vitro and in vivo, suggesting that 2B4-CD48 interactions protects CD8 T cells from NK cell-mediated killing during viral infection. ${ }^{43}$ Also, NK cell-deficient ( $\mathrm{Nfil}^{-/-}$) mice or NK celldepleted wild-type mice infected with LCMV displayed an increased virus-specific CD8 T cell response, resulting in earlier virus control. In this setting, LCMV-specific T cells expressed NKG2D ligands, whereas NKG2D was up-regulated and 2B4 was down-regulated on NK cells, resulting in a negative regulation of T cell proliferation and IFN $-\gamma$ production in a NKG2D and perforin-dependent manner. ${ }^{44}$ The use of NCR1-deficient mice and transfer experiments showed that NK cells can directly kill virus-activated CD4 and CD8 T cells during chronic LCMV infection, in a NKp46-depedent manner. In the absence of NCR1 (that encodes for NKp46), CD8 T cells exhibited an enhanced effector response, which resulted in faster viral clearance accompanied by severe immunopathology, supporting the notion that the regulatory activity of NK cells is important to limit T cell-mediated damage to the host. ${ }^{45}$ More recently, it was observed that LCMV-activated NK cells can kill all activated CD4 subsets and CD8 T cell in vitro and in vivo in a perforin-dependent manner, yet the different $T$ cell subsets showed a hierarchy of sensitivity, being. Th0 and Th1 cells the most resistant and regulatory T cell (Treg), Th17, Th2, and CD8 T cells the more sensitive to NK cell cytotoxicity. ${ }^{46}$

Other viral infections also provided evidence for a regulatory role of NK cells. Depletion of NK cells in murine cytomegalovirus (MCMV)-infected mice resulted in enhanced IFN- $\gamma$ production by CD4 and CD8 T cells, and CD8 T cell proliferation. ${ }^{42}$ Consistent with an immunoregulatory role, NK cells from Noé mice (mice with a loss of function mutation in NCR1 gene that display over-expression of the Helios transcription factor) display an hyperreactive phenotype and the ability to inhibit IFN- $\gamma$ production by CD4 and CD8 T cells in MCMV-infected mice and the generation of a fully protective memory $\mathrm{T}$ cell response to the intracellular bacteria Listeria monocytogenes. ${ }^{47}$ Remarkably, MCMV infection in mice recapitulates many aspects of infection of humans with $\mathrm{CMV}^{48}$ In both species, infection with the corresponding CMV promotes the expansion of NK cells with features of cells of the adaptive immune response characterized by the expression of $\mathrm{Ly} 49 \mathrm{H}$ in mice and CD94/NKG2C in humans. ${ }^{49}$ However, whether NKG2C+ human NK cells can display a putative regulatory function during $\mathrm{CMV}$ infection remains unknown. 
Additional transfer experiments demonstrated that NK cells activated by LCMV, Pichinde virus, mouse hepatitis virus, or poly I:C could eliminate LCMV-activated CD4 T cells. Reciprocally, LCMV-activated NK cells were able to lyse CD4 T cells activated by infections with LCMV, Pichinde virus, MCMV, mouse hepatitis virus, or vaccinia virus. ${ }^{50}$ These results provided evidence that the killing of activated CD4 T cells by activated NK cell during viral infections is a widespread phenomenon. In these settings, activated CD4 T cells were significantly more susceptible to NK cell-mediated elimination than activated CD8 $T$ cells, presumably because of higher expression of CD48 on activated CD8 T cells. However, as CD4 T cells provide help to generate CD8 T cell responses to $\mathrm{LCMV}$, NK cells also indirectly can regulate the magnitude of CD8 T cell responses through the elimination of such CD4 T cells. ${ }^{50}$ Nevertheless, the capacity of NK cells to inhibit T cell responses seems to relate to the virus ability to induce NK cell activation. NK cells become poorly activated by vaccinia virus resulting in higher expression of NKG2A and lower expression of activation markers and effector molecules compared with the LCMV-activated NK cells, and these NK cells exhibit a reduced ability to constrain CD4 T cell responses. ${ }^{51}$

Depending on the infective dose and the pathogen, NK cellmediated regulation of $\mathrm{T}$ cell activity may be beneficial or detrimental to the host. For example, NK cell depletion has minimal effect on $\mathrm{T}$ cell-mediated immunity or immunopathology in mice infected with low doses of LCMV-clone 13, a strain that generates chronic disease, or acutely infected with LCMV-Armstong, a strain that is spontaneously cleared after acute infection. ${ }^{50,52}$ In contrast, infection of mice with high doses of LCMV-clone13, resulted in the accumulation of exhausted $T$ cells that contributed to viral persistence but prevented fatal pathology, and this effect was not observed in NK cell-depleted mice. ${ }^{50,52}$ Conversely, at medium doses of infection, NK cells fostered $\mathrm{T}$ cell-mediated disease. Therefore, NK cells seem to act as a rheostat that regulates the magnitude of antiviral $T$ cell responses and critically affect the outcome of the infection, which may result in resolution, chronicity, or immunopathology. ${ }^{50}$

Type I IFN are key cytokines rapidly induced during viral infections that, in LCMV-activated antigen-specific T cells, can induce the expression of ligands for NK cell inhibitory receptors such as classical MHC-I molecules and Qa-1 $b^{53}$ and restrict the expression of NKp46 ligands, ${ }^{54}$ protecting activated T cells from NK cell-mediated perforindependent lysis. Also, type I IFN, by suppressing the up-regulation of MCMV-induced NKG2D ligands on NK cells, protects NK cells from fratricide through NKG2D and perforin-mediated elimination. ${ }^{55}$ In humans, IFN- $\beta$ signaling in CD4 T cells induces HLA-E expression, rescuing them from NK cell-mediated elimination. ${ }^{56}$ Thus, type I IFN signaling triggered during viral infections can attenuate $T$ cell susceptibility to NK cell-mediated elimination, and this phenomenon may contribute to the adequate expansion of effector $T$ cells required to control the viral infections.

As mentioned above, NK cells can induce death receptor-mediated apoptosis through expression of TRAIL and engagement of TRAIL receptors DR4 (TRAIL-R1) or DR5 (TRAIL-R2) on target cells. ${ }^{16}$ During chronic MCMV infection of BALB/c mice, TRAIL ${ }^{+} \mathrm{NK}$ cells accumulate in the salivary glands, whereas activated CD4 T cells express NKG2D ligands and TRAIL-R2, making them susceptible to NKG2D-mediated recognition and TRAIL-dependent deletion by NK cells. ${ }^{57}$ Remarkably, depletion of NK cells resulted in a faster resolution of the infection but the resulting magnified inflammatory response resulted in an autoimmune disease similar to human Sjogren's syndrome. ${ }^{57}$ In humans, a similar mechanism aimed at controlling the magnitude of the antiviral CD8 T cell response seems to operate in patients with chronic hepatitis $B$ virus (HBV) infection. Accordingly, in chronic HBV-infected patients, high expression of TRAIL was detected on liver NK cells, whereas high expression of TRAIL-R2 was observed on HBV-specific CD8 T cells, and this last population was further enriched in the liver and susceptible to caspase-8-mediated apoptosis. ${ }^{58}$ Recently, a novel nonapoptotic pathway for TRAIL signaling on NK cells was described that involves an IL-15-induced TRAIL-mediated enhancement of granzyme B production by NK cells that results in diminished CD8 T cell responses due to T cell elimination in LCMV-infected mice. ${ }^{59}$

In addition to their ability to directly eliminate T cells, NK cells were shown to also indirectly suppress $T$ cell responses through the secretion of IL-10. ${ }^{60,61}$ Compared with the NK cells from healthy donors, NK cells from individuals chronically infected with hepatitis $\mathrm{C}$ virus (HCV) produce larger amounts of IL-10,62,63 which in turn could inhibit DC maturation. ${ }^{62}$ Likewise, NK cells from patients chronically infected with HBV produce larger amounts of IL-10 than healthy donors and monocytes exposed to HBV induced the differentiation of IL-10-producing NK cells, which inhibited HBV-specific-T cell proliferation and IFN- $\gamma$ production in a IL-10-dependent manner. ${ }^{64}$

NK cells can also indirectly regulate $B$ cell responses against LCMV through perforin-dependent elimination of follicular helper CD4 T cells (Tfh), and a subsequent inhibition of germinal center responses and antibody generation. ${ }^{65,66}$

\subsection{Regulatory functions of NK cells during bacterial and parasite infections}

Evidence about a regulatory function of NK cells during bacterial and parasite infections are less abundant. In mice, NK cells are an early source of IL-10 during systemic infections with Toxoplasma gondii or Yersinia pestis. Systemic but not localized infections induced IL-12 production by DCs, which subsequently triggered IL-10 production by NK cells that limited further production of IL-12. ${ }^{67}$ In contrast, during $L$. monocytogenes infection, IL-10 production by NK cells is driven by IL-18 in an IL-12-independent way, ${ }^{68}$ arguing that at least 2 different pathways can induce IL-10 secretion by NK cells. Additionally, NK cell production of IL-10 during Leishmania donovani69 and $L$. monocytogenes ${ }^{70}$ infection results in inhibition of protective immunity, whereas during infection with MCMV, NK cell-derived IL-10 regulates the magnitude of endogenous CD8 T cell responses and protects the host from CD8 T cell-dependent immunopathology. ${ }^{71}$

As with viruses, NK cells can also indirectly regulate B cell responses against bacteria through a similar mechanism that involves the limitation of antibody production due to the elimination of Tfh cells that inhibit the germinal center reaction during L. monocytogenes, ${ }^{65}$ and also during immunization. ${ }^{72}$ 
TA B LE 1 Regulation of immune responses by NK cells during infections

\begin{tabular}{|c|c|c|c|c|}
\hline \multirow[t]{3}{*}{ Mouse } & - LCMV & $\begin{array}{l}\text { - CD8 T cells } \\
\text { - CD4 T cells } \\
\text { - CD4 and CD8 T cells } \\
\text { - CD8 T cells and APC } \\
\text { - NK cells } \\
\text { - CD8 T cells } \\
\text { - CD4 T cells, Tfh and B cells }\end{array}$ & $\begin{array}{l}\text { - NKG2D, 2B4 or NKp46, and perforin } \\
\text { - N/D } \\
\text { - Type I IFNs, NKp46, and perforin } \\
\text { - N/D } \\
\text { - Type I IFN, NKG2D, and perforin } \\
\text { - TRAIL, IL-15, IFN- } \gamma \text {, granzyme B, and perforin } \\
\text { - Perforin }\end{array}$ & $\begin{array}{l}43-45,53 \\
80 \\
50,54 \\
52 \\
55 \\
59 \\
65,66\end{array}$ \\
\hline & - Toxoplasma gondii & $-\mathrm{DCs}$ & $-I L-10$ & 67 \\
\hline & -Listeria monocytogenes & - Inflammatory myeloid cells & $-I L-10$ & 68,70 \\
\hline Human & - Chronic HBV & $\begin{array}{l}\text { - CD8 T cells } \\
\text { - T cells }\end{array}$ & $\begin{array}{l}\text { - TRAIL and caspase-8 } \\
\text { - IL-10 }\end{array}$ & $\begin{array}{l}58 \\
64\end{array}$ \\
\hline
\end{tabular}

LCMV, lymphocytic choriomeningitis virus; N/D, not determined; IFNs, interferons; APC, antigen presenting cells; Tfh, follicular helper CD4 T cells; MCMV, murine cytomegalovirus; DCs, dendritic cells; $\mathrm{HCV}$, hepatitis C virus; $\mathrm{HBV}$, hepatitis B virus.

TAB LE 2 Regulation of immune responses by NK cells during autoimmunity

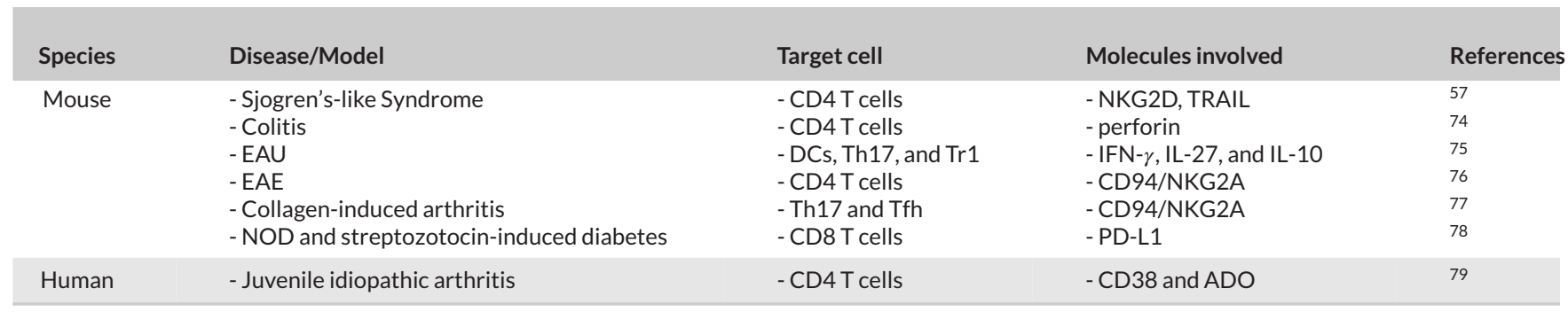

EAU, experimental autoimmune uveitis; Th17, T helper 17 cells; Tr1, type 1 regulatory cells; IFN, interferon; EAE, experimental autoimmune encephalomyelitis; Tfh, follicular helper CD4 T cells; PD-L1, programmed death-ligand 1; ADO, adenosine.

\section{I REGULATORY FUNCTIONS OF NK CELLS DURING AUTOIMMUNITY AND INFLAMMATION}

Viral infections and the resulting antiviral immunity (in which NK cells play an important role) are associated with the development of several systemic autoimmune diseases. ${ }^{73}$ Paradoxically, in a model of induced colitis, NK cells ameliorated the severity of the disease through perforin-dependent elimination of effector CD4 T cells. $^{74}$ Also, during the development of experimental autoimmune uveitis (EAU), IFN- $\gamma$ derived from NK cells was sufficient to reduce the severity of the disease by suppressing the development of Th17 responses though a circuit that involved a NK cell-derived IFN- $\gamma$ induction of IL-27 production by DCs, which potentiated IFN- $\gamma$ production by NK cells and induced IL-10 production by both cells, and contributed to inhibit pathogenic Th17 responses. ${ }^{75}$ Therefore, NK cells can suppress immunopathology in different models of autoimmune disorders, which highlights their dual role as effector cells and as regulatory cells of the immune response against pathogens.

Additional evidence unraveled a role of $\mathrm{MHC}-\mathrm{I}$ class lb molecule Qa-1, which is recognized by the NK cell inhibitory receptor CD94/NKG2A, in T cell protection from NK cell-mediated cytotoxicity. During the development of experimental autoimmune encephalomyelitis (EAE), the interaction between Qa-1 on CD4 T cells with CD94/NKG2A on NK cells prevented NK cell-dependent $T$ cell elimination and contributed to amplify the expansion of autoreactive CD4 T cells, whereas antibody-mediated blockade of Qa-1 or adoptive transfer of autoreactive Qa-1-1- CD4 T cells attenuated the severity of the disease. ${ }^{76}$ In addition, in mice with collagen-induced arthritis (which resembles human rheumatoid arthritis in some aspects) where pathology is mediated by Th17 cells and Th cells that facilitate the expansion of autoreactive B cells, blockade of NKG2A interaction with Qa-1 fostered NK cell-mediated elimination of Th17 and Tfh cells in vitro and ameliorated arthritis progression in vivo. ${ }^{77}$ Moreover, another subset of regulatory NK cells, characterized by the expression 


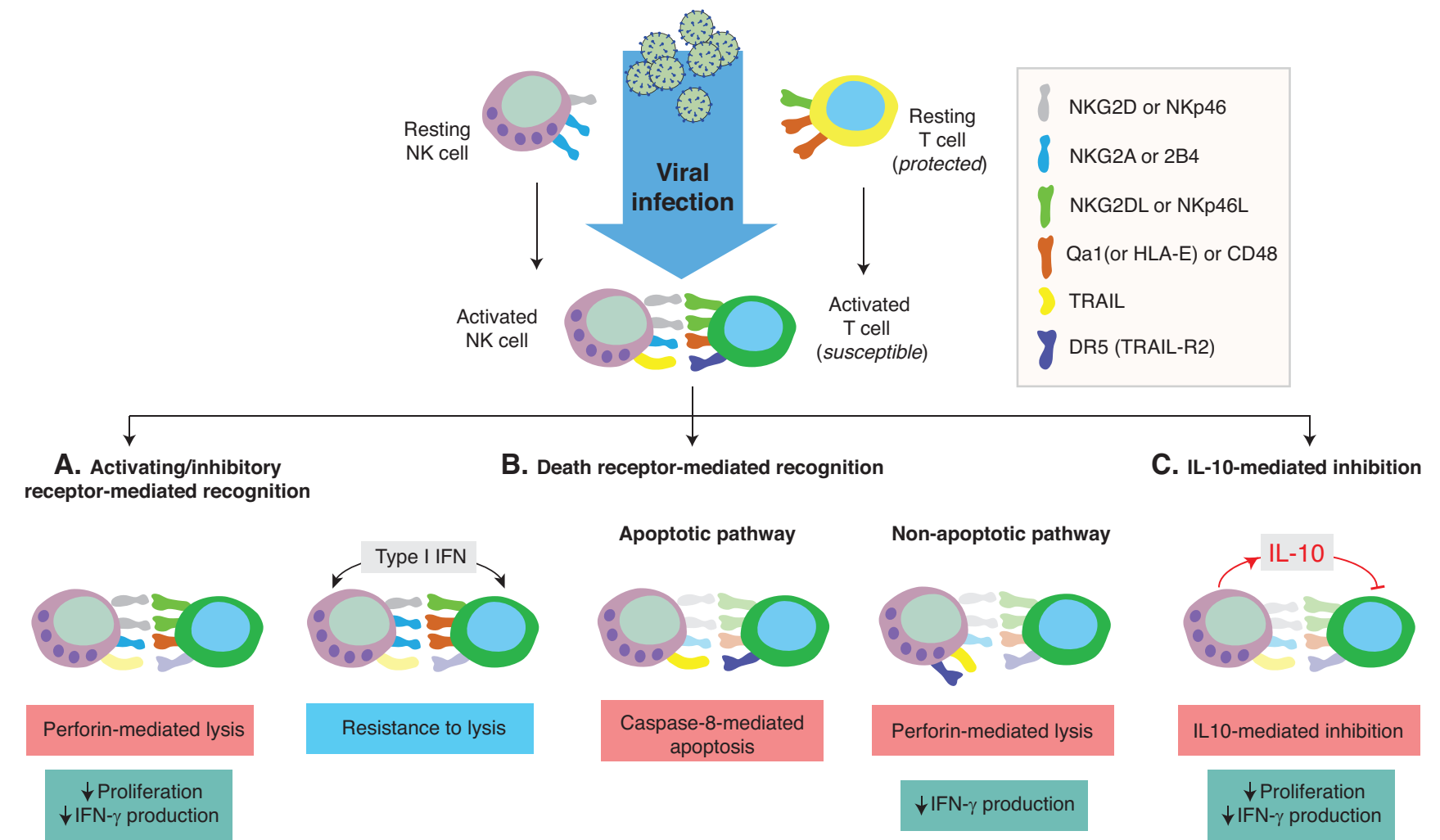

FIGURE 1 Direct inhibition of T cell responses by NK cells during viral infections. Following viral infections, activated NK cells can inhibit $T$ cell responses through different mechanisms. (A) Activating/inhibitory receptor-mediated recognition: activated NK cells show an enhanced expression of activating receptors and reduced expression of inhibitory receptors, whereas activated T cells show an augmented expression of ligands for activating receptors, which makes them susceptible to NK cell-mediated recognition and perforin-mediated lysis. Type I IFN signaling on both cells, through modulation of the expression of such receptors and ligands, can protect activated T cells from NK cell-mediated lysis. (B) Death receptor-mediated recognition. Caspase 8-mediated apoptosis can be induced on DR5-expressing T cells upon engagement of TRAIL on NK cells (Apoptotic pathway); also, TRAIL signaling on NK cells can enhance the production of IL-15-induced granzyme B, which results in increased perforin-mediated lysis of T cells (Non-apoptotic pathway), and (C) IL-10-mediated inhibition: NK cell-derived IL-10 can directly inhibit CD4 and CD8 T cell responses

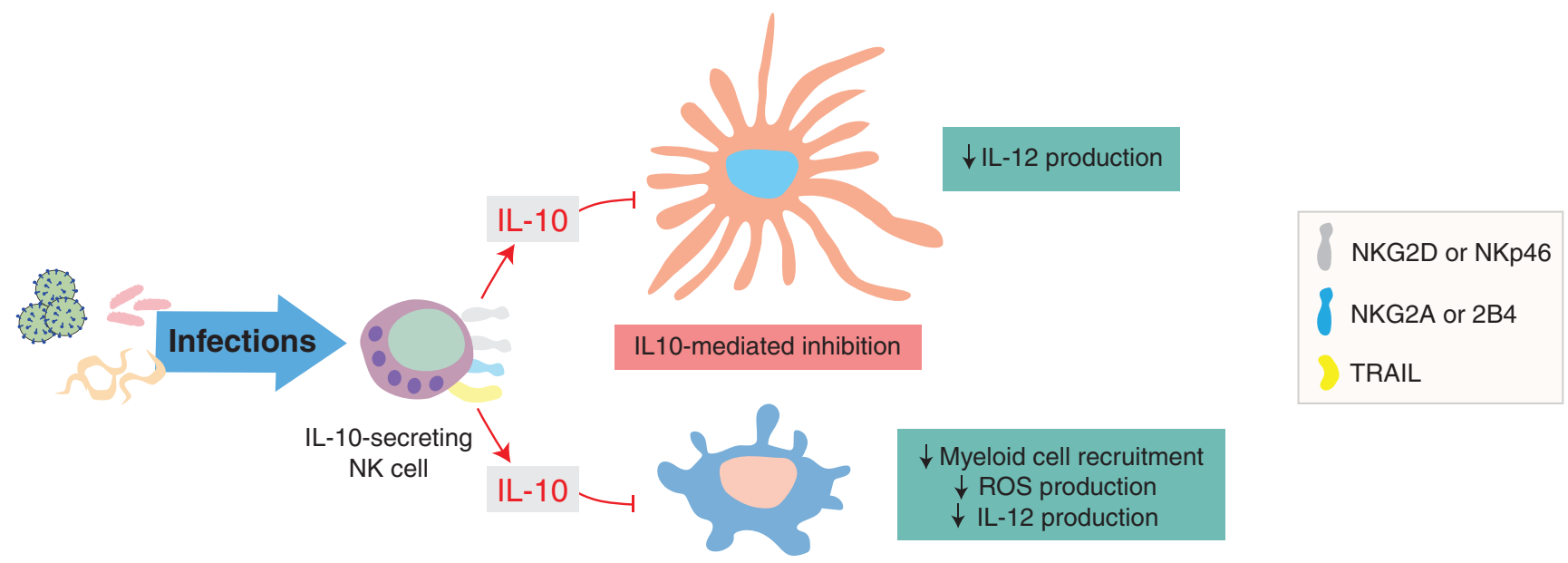

FIG URE 2 Regulation of innate responses by NK cells during infections. Following some systemic viral, bacterial or parasite infections, NK cells can secrete IL-10 which inhibits IL-12 production by dendritic cells (DCs). Moreover, NK cell-derived IL-10 inhibits the recruitment and activation of myeloid cells 
TA B LE 3 Regulation of immune responses by NK cells in cancer

\begin{tabular}{|c|c|c|c|c|}
\hline Species & Cell line/Tumor & Target cell & Molecules involved & References \\
\hline Mouse & $\begin{array}{l}\text { - RMA and B16F10 } \\
\text { - MC57 } \\
\text { - B16F10 and CT26 } \\
\text { - B16F10 } \\
\text { - LN7 }\end{array}$ & $\begin{array}{l}\text { - DCs } \\
\text { - DCs } \\
\text { - DCs } \\
\text { - CD8 T cells } \\
\text { - Treg }\end{array}$ & $\begin{array}{l}\text {-TRAIL and arginase-1 } \\
\text { - PD-L1 } \\
\text { - PD-L1 and IL-18 } \\
\text { - NKG2D and perforin } \\
\text { - CCL22 }\end{array}$ & $\begin{array}{c}83,84 \\
85 \\
86 \\
88 \\
89\end{array}$ \\
\hline Human & $\begin{array}{l}\text { - Serous ovarian cancer } \\
\text { - NSCLC } \\
\text { - Breast cancer } \\
\text { - Melanoma }\end{array}$ & $\begin{array}{l}\text { - T cells } \\
\text { - CD4 T cells } \\
\text { - CD4 T cells } \\
\text { - N/D }\end{array}$ & $\begin{array}{l}\text { - NKp46 } \\
\text { - NKp46 } \\
\text { - CD73 and IL-10 } \\
\text { - CD38 }\end{array}$ & $\begin{array}{c}90 \\
91 \\
92,93 \\
95\end{array}$ \\
\hline
\end{tabular}

RMA, T cell lymphoma cell line; B16F10, melanoma cell line; iNOS, inducible nitric oxide synthase; MC57, mouse fibrosarcoma cell line; CT26, colon carcinoma cell line; LN7, metastatic cell line of Lewis lung carcinoma; Treg, regulatory T cell; NSCLC, non-small cell lung cancer; N/D, not determined.

of CD117 (c-Kit) and programmed death-ligand 1 (PD-L1) and that could kill activated CD8 T cells (but not CD4 T cells) through PDL1-recognition in vitro and in vivo, was described. ${ }^{78}$ These CD117 $^{+}$ NK cells could be generated in vitro upon IL-18 stimulation of naïve CD117- NK cells and were detected in spleens and lymph nodes of healthy mice and, at lower frequencies, in NOD mice. Remarkably, adoptive transfer of IL-18-stimulated NK cells (presumably containing an increased abundance of these $\mathrm{CD} 117^{+} \mathrm{NK}$ cells) reduced the severity of streptozotocin-induced diabetes in mice in a PD-L1-dependent manner. ${ }^{78}$ Also, $\mathrm{CD} 38^{+} \mathrm{CD} 56^{\text {bright }} \mathrm{NK}$ cells can inhibit CD4 T cell proliferation through the production of adenosine, whereas CD56 bright NK cells from the synovial fluid of patients with juvenile idiopathic arthritis express lower amounts of CD38 and fail to inhibit CD4 T cell proliferation, confirming the role of CD38 expressed by NK cells in the regulation of $\mathrm{T}$ cell proliferation. ${ }^{79}$

Taken together, these studies strongly point to the existence of NK cells with a regulatory role that, through different and perhaps overlapping and/or redundant mechanisms, contribute to an optimal sizing of the magnitude of the $T$ cell-mediated immune response against pathogens, limiting the damage to self-tissues and whose interference may tilt the balance toward immunopathology in different conditions (summarized in Tables 1 and 2 and Figs. 1 and 2).

\section{4 | REGULATORY FUNCTIONS OF NK CELLS IN ANTITUMOR IMMUNITY}

The tumor microenvironment induces multiple phenotypic and functional modifications on immune cells. Tumor-infiltrating CD8 T cells display an exhausted phenotype, transcriptionally similar to exhausted CD8 T cells from chronic LCMV-infected mice. ${ }^{81}$ Also, NK cells infiltrating tumors exhibit an altered phenotype and are often dysfunctional. ${ }^{82}$ Despite several studies that unraveled the regulatory functions of NK cells during infections and that were discussed above, less is known about regulatory NK cells during tumor progression. Early work using a mouse syngeneic model of RMA lymphoma revealed that the depletion of NK cells induced enhanced antigen presentation by DCs that resulted in increased numbers of tumor-specific

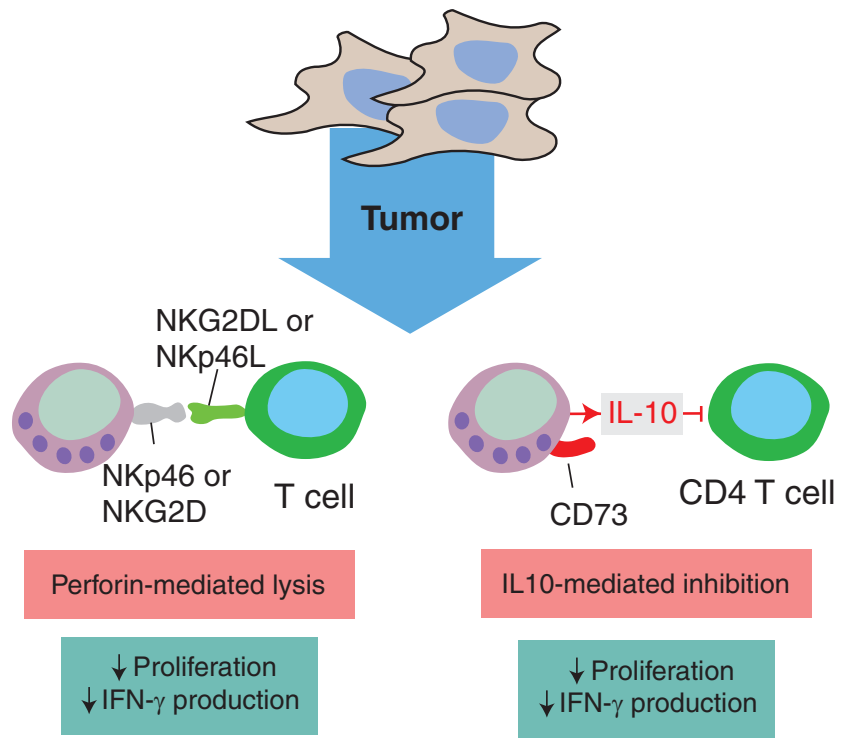

FIGURE 3 Direct inhibition of T cells by NK cells during antitumor immune responses. Tumor progression leads to modifications in NK cell phenotype and function, making them able to directly inhibit $T$ cell responses. NK cells can kill antigen-specific CD8 T cells in a NKG2D and perforin-dependent manner and can inhibit $T$ cell proliferation and effector functions in an NKp46-dependent manner. Also, a population of IL-10-producing NK cells (characterized by the expression of CD73) is able to inhibit CD4 T cell proliferation and IFN- $\gamma$ production in an IL-10-dependent manner

CD8 T cells, enhanced tumor-specific lysis and IFN- $\gamma$ production. ${ }^{83}$ It was later observed that the interaction between TRAIL on NK cells and TRAIL-R2 on DCs induced the expression of several immunoregulatory molecules such as IL-10, iNOS, and arginase-1 in DCs, which resulted in arginase-1-dependent inhibition of the DCs cross-priming ability of CD8 T cells against tumor-associated antigens. ${ }^{84}$

Consistent with these results, we observed that following subcutaneous implantation of immunogenic MC57 tumor cells transduced to express the model antigenic peptide SIYRYYGL (SIY), NK cell depletion led to a significantly higher frequency of spontaneously activated SIY-specific CD8 T cells, with enhanced IFN- $\gamma$ production and cytotoxic capability. ${ }^{85}$ Furthermore, NK cells also constrained the expansion of an effector memory CD8 T cell population, which 


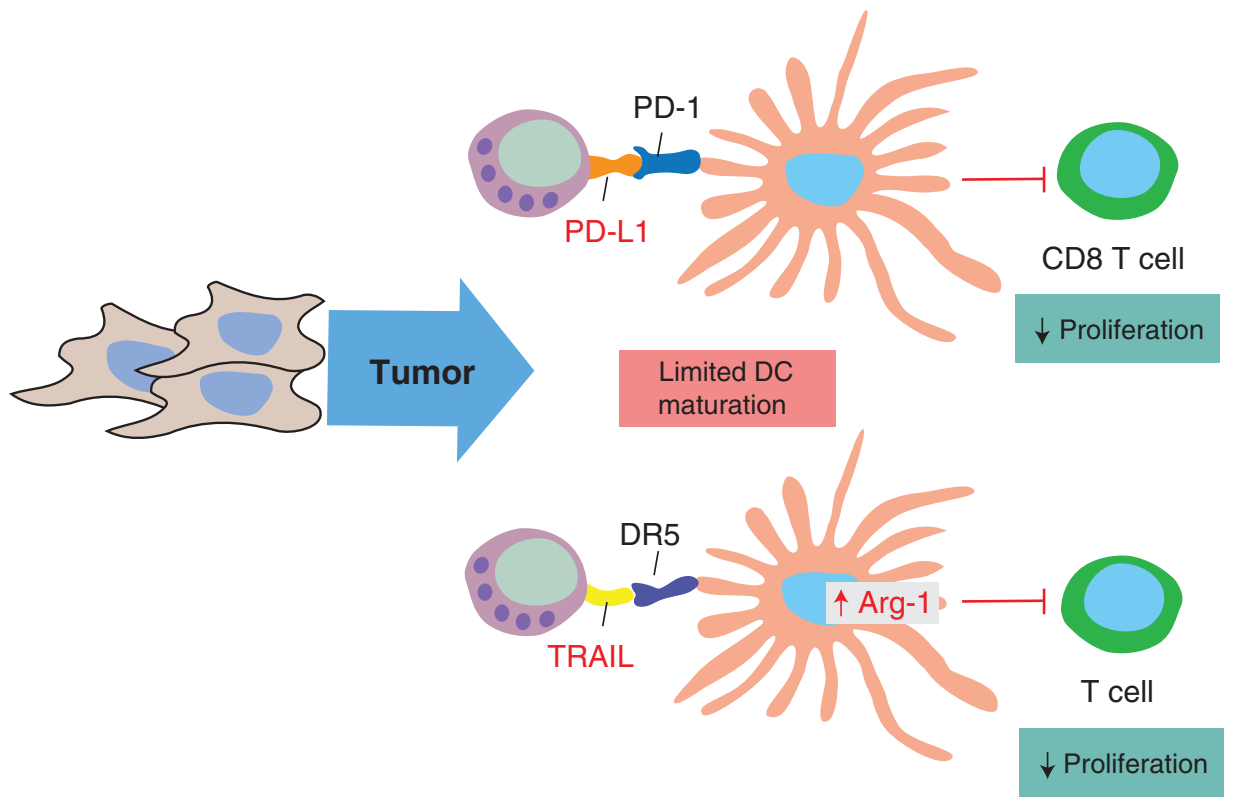

FIGURE 4 Indirect inhibition of antitumor T cell responses by NK cells through regulation of DCs maturation and/or cross presentation. During tumor progression NK cells can modulate DCs activation, resulting in attenuated T cell priming. The interaction between TRAIL on NK cells and DR5 on DCs induces the expression of arginase-1 (Arg-1) on DCs, which inhibits the cross-presentation of tumor antigens, and results in reduced cross-priming of CD8 T cells. Tumor-infiltrating NK cells can also express PD-L1, which through interaction with PD-1 expressed on DCs, limits DCs maturation, resulting in diminished CD8 T cell priming

resulted in diminished recall response and reduced tumor control after a secondary tumor challenge with the poorly immunogenic B16.SIY tumor. ${ }^{85}$ Analysis of tumor-infiltrating and tumor-draining lymph node NK cells showed an up-regulated expression of the inhibitory molecule PD-L1 compared with peripheral blood NK cells from the same animals or from tumor-free mice. Mechanistically, DCs from tumor-bearing mice exhibited PD-1 expression and NK cell inhibited DCs maturation through PD-L1, explaining their reduced ability to induce CD8 T cell priming. Together, these data suggest that PD-L1 ${ }^{\text {hi }}$ NK cells that emerge during tumor growth can directly regulate DCs maturation resulting in a reduced ability to support CD8 T cell priming and recall responses against tumor antigens. ${ }^{85}$ In another study, CD $117^{+}$PD-L1 ${ }^{+}$NK cells were shown to be induced by B16 and CT26-derived IL-18, and adoptive transfer of these NK cells resulted in reduced numbers of DCs and promoted the growth of both tumors in a PD-L1 dependent manner. ${ }^{86}$ The presence of PD-L1+ NK cells was also described in patients with acute myeloid leukemia, and anti-PD-L1 antibodies promoted enhanced NK cell effector functions and a better control of PD-L1- tumors in a PD-1 independent-manner. ${ }^{87}$

Additionally, after immunization of OT1 mice with OVA, NK cells could kill antigen-specific CD8 T cells in a NKG2D and perforindependent manner, whereas immunization in the absence of NK cells resulted in a higher frequency of OT1 CD8 T cells and an enhanced memory response that resulted in an improved tumor control when immunized mice were challenged with B16.OVA tumor cells. ${ }^{88} \mathrm{~A}$ different and more indirect mechanism for regulatory NK cells was described in a model of Lewis Lung carcinoma where NK cells induced Treg recruitment through CCL22 secretion, which in turn could contribute to immunoregulation. ${ }^{89}$
In humans, the presence of a population of $\mathrm{CD}^{-} \mathrm{CD} 56^{+}$cells in cultures of tumor-infiltrating lymphocytes (TIL) from patients with primary high-grade serous ovarian cancer was associated with reduced numbers of CD4 and CD8 T cells. These regulatory CD3- CD56 ${ }^{+}$ cells showed an expression profile overlapping with those of NK cells and other ILCs, expressed NKG2D, NKp30 and NKp46, and suppressed TIL proliferation and TNF- $\alpha$ and IFN- $\gamma$ production in an NKp46-dependent manner Moreover, the presence of these regulatory $\mathrm{CD}^{-} \mathrm{CD}^{-} 6^{+}$cells in TIL cultures correlated with a reduction in the time to relapse. ${ }^{90}$ In addition, a higher frequency of a population of circulating $\mathrm{NKp} 46^{+} \mathrm{CD} 56^{\mathrm{dim}} \mathrm{CD} 16^{+} \mathrm{NK}$ cells was associated with a decreased overall survival in patients with non-small cell lung cancer (NSCLC), and NKp46 blockade enhanced IFN- $\gamma$ production by tumor antigen-specific CD4 T cells in cultures of patientderived PBMC. ${ }^{91}$

A recent study shows that tumor-infiltrating NK cells from breast cancer patients display an increased expression of the $5^{\prime}$ ectonucleotidase CD73, accompanied by up-regulation of other immune checkpoint receptors such as LAG-3, VISTA, PD-1, and PD-L1. ${ }^{92}$ The induction of enzymatically active CD73 was dependent on 4-1BBL recognition on tumor cells and $\mathrm{CD}_{73}{ }^{+} \mathrm{NK}$ cells were able to inhibit CD4 T cell proliferation and IFN- $\gamma$ production, but the inhibition was not dependent on the production of extracellular adenosine by CD73 activity. Instead, STAT3-mediated IL-10 production by these CD73+ NK cells accounted for the suppressive effect. ${ }^{92}$ Consistent with these results, IL-10-producing NK cells were detected in the circulation of a different cohort of breast cancer patients. ${ }^{93}$ Another study demonstrated that, in melanoma patients, a high frequency of circulating CD56 bright $\mathrm{NK}$ cells with an altered phenotype, including increased 
expression of CD38 (another ectoenzyme involved in the noncanonical production of adenosine ${ }^{94}$ ) is associated with a worsened outcome. ${ }^{95}$

Together, these studies start to outline the role of NK cells in the direct and indirect mechanisms involved in regulation of antitumor adaptive immune responses in mouse and humans (summarized in Table 3 and Figs. 3 and 4).

\section{5 | CONCLUDING REMARKS}

NK cells can provide multiple levels of inhibition for $T$ cell responses, including indirect inhibition through targeting of DCs, direct lysis of activated $\mathrm{T}$ cells or regulation of $\mathrm{T}$ cell effector function through the secretion of immunoregulatory cytokines or the expression of discrete cell surface receptors not involved in direct target cell lysis. Moreover, the regulation of CD4 T cell responses has also implications on the generation of B cell immunity. Regardless of the ultimate mechanism used to constrain the adaptive immune response, recently elucidated regulatory functions of NK cells may constitute a mechanism to prevent overactivation of adaptive immune cells during chronic infections that otherwise may lead to immunopathology. However, some tumors may hijack these physiologic regulatory functions, coopting their mechanism to promote immune tolerance and tumor escape. Therefore, the elucidation of phenotypic markers that define NK cells with regulatory functions may lead to the development of novel modalities of immunotherapy in which a timely depletion of NK cells with regulatory functions may improve the efficacy of current immunotherapies. Also, a more comprehensive elucidation of the mechanisms that drive the generation and action such NK cells with regulatory functions will probably contribute to the development of strategies aimed at the manipulation of their expansion and effector functions in different pathologic conditions with the ultimate goal of appropriately sizing an optimal adaptive immune responses in cancer and chronic viral infections, and, on the other hand, promote tolerance in transplants and autoimmune disorders.

\section{AUTHORSHIP}

N.W.Z. and M.B.F. wrote the article. C.I.D. prepared the tables. M.B.F. prepared the figures. N.W.Z., C.I.D. and M.B.F. edited the manuscript and approved it for publication.

\section{ACKNOWLEDGMENTS}

We thank the National Agency for Promotion of Science and Technology from Argentina (ANPCYT) and the National Research Council of Argentina (CONICET) for providing funding for research. C.I.D. M.B.F. and N.W.Z. are members of the Researcher Career of CONICET.

\section{DISCLOSURES}

The authors declare no conflicts of interest.
ORCID

Norberto W. Zwirner iD https://orcid.org/0000-0001-7098-359X

Carolina I. Domaica (iD https://orcid.org/0000-0001-9596-4889

Mercedes B. Fuertes (iD https://orcid.org/0000-0002-2734-1468

\section{REFERENCES}

1. Mace EM, Orange JS. Emerging insights into human health and NK cell biology from the study of NK cell deficiencies. Immunol Rev. 2019;287:202-225.

2. Newman KC, Riley EM. Whatever turns you on: accessory-celldependent activation of NK cells by pathogens. Nat Rev. 2007;7: 279-291.

3. Lanier LL. Evolutionary struggles between NK cells and viruses. Nat Rev. 2008;8:259-268.

4. Morvan MG, Lanier LL. NK cells and cancer: you can teach innate cells new tricks. Nat Rev Cancer. 2016;16:7-19.

5. Shifrin N, Raulet DH, Ardolino M. NK cell self tolerance, responsiveness and missing self recognition. Semin Immunol. 2014;26:138-144.

6. Imai K, Matsuyama S, Miyake S, et al. Natural cytotoxic activity of peripheral-blood lymphocytes and cancer incidence: an 11-year follow-up study of a general population. Lancet. 2000;356:1795-1799.

7. Martin-Fontecha A, Thomsen LL, Brett S, et al. Induced recruitment of NK cells to lymph nodes provides IFN-gamma for $\mathrm{T}(\mathrm{H}) 1$ priming. Nat Immunol. 2004;5:1260-1265.

8. Mailliard RB, Alber SM, Shen H, et al. IL-18-induced CD83+CCR7+ NK helper cells. J Exp Med. 2005;202:941-953.

9. Wong JL, Berk E, Edwards RP, et al. IL-18-primed helper NK cells collaborate with dendritic cells to promote recruitment of effector CD8+ T cells to the tumor microenvironment. Cancer Res. 2013;73: 4653-4662.

10. Carrega P, Ferlazzo G. Natural killer cell distribution and trafficking in human tissues. Front Immunol. 2012;3:347.

11. Melsen JE, Lugthart G, Lankester AC, et al. Human circulating and tissue-resident CD56(bright) natural killer cell populations. Front Immunol. 2016;7:262

12. Vivier E, Artis D, Colonna M, et al. Innate lymphoid cells: 10 years on. Cell. 2018;174:1054-1066.

13. Klose CSN, Artis D. Innate lymphoid cells as regulators of immunity, inflammation and tissue homeostasis. Nat Immunol. 2016;17:765-774.

14. Yang C, Siebert JR, Burns R, et al. Heterogeneity of human bone marrow and blood natural killer cells defined by single-cell transcriptome. Nat Commun. 2019;10:3931.

15. Campbell JJ, Qin S, Unutmaz D, et al. Unique subpopulations of CD56+ NK and NK-T peripheral blood lymphocytes identified by chemokine receptor expression repertoire. J Immunol. 2001;166:6477-6482.

16. Prager I, Watzl C. Mechanisms of natural killer cell-mediated cellular cytotoxicity. J Leukoc Biol. 2019;105:1319-1329.

17. Zwirner NW, Domaica $\mathrm{Cl}$. Cytokine regulation of natural killer cell effector functions. Biofactors. 2010;36:274-288.

18. Freud AG, Mundy-Bosse BL, Yu J, et al. The broad spectrum of human natural killer cell diversity. Immunity. 2017;47:820-833.

19. Caligiuri MA. Human natural killer cells. Blood. 2008;112:461-469.

20. Strowig T, Brilot F, Munz C. Noncytotoxic functions of NK cells: direct pathogen restriction and assistance to adaptive immunity. J Immunol. 2008;180:7785-7791.

21. Moretta A, Marcenaro E, Parolini S, et al. NK cells at the interface between innate and adaptive immunity. Cell Death Differ. 2008;15:226-233.

22. Michel T, Poli A, Cuapio A, et al. Human CD56bright NK cells: an update. J Immunol. 2016;196:2923-2931. 
23. Ferlazzo G, Thomas D, Lin SL, et al. The abundant NK cells in human secondary lymphoid tissues require activation to express killer cell Iglike receptors and become cytolytic. J Immunol. 2004;172:1455-1462.

24. Ferlazzo G, Pack M, Thomas D, et al. Distinct roles of IL-12 and IL-15 in human natural killer cell activation by dendritic cells from secondary lymphoid organs. Proc Natl Acad Sci USA. 2004;101:16606-16611.

25. Chan A, Hong DL, Atzberger A, et al. CD56bright human NK cells differentiate into CD56dim cells: role of contact with peripheral fibroblasts. J Immunol. 2007;179:89-94.

26. Romagnani C, Juelke K, Falco M, et al. CD56brightCD16- killer lg-like receptor- NK cells display longer telomeres and acquire features of CD56dim NK cells upon activation. J Immunol. 2007;178:4947-4955.

27. Luetke-Eversloh M, Killig M, Romagnani C. Signatures of human NK cell development and terminal differentiation. Front Immunol. 2013;4:499.

28. Domaica $\mathrm{Cl}$, Fuertes $\mathrm{MB}$, Uriarte I, et al. Human natural killer cell maturation defect supports in vivo CD56(bright) to CD56(dim) lineage development. PLoS One. 2012;7:e51677.

29. Caldirola MS, Rodriguez Broggi MG, Gaillard MI, et al. Primary immunodeficiencies unravel the role of IL-2/CD25/STAT5b in human natural killer cell maturation. Front Immunol. 2018;9:1429.

30. Vivier E, Tomasello E, Baratin M, et al. Functions of natural killer cells. Nat Immunol. 2008;9:503-10.

31. Kim S, lizuka K, Kang H-SP, et al. In vivo developmental stages in murine natural killer cell maturation. Nat Immunol. 2002;3:523-528.

32. Hayakawa Y, Huntington ND, Nutt SL, et al. Functional subsets of mouse natural killer cells. Immunol Rev. 2006;214:47-55.

33. Nunez SY, Ziblat A, Secchiari F, et al. Human M2 Macrophages Limit NK Cell Effector Functions through Secretion of TGF- $\beta$ and engagement of CD85j. J Immunol. 2018;200:1008-1015.

34. Girart MV, Fuertes MB, Domaica Cl, et al. Engagement of TLR3, TLR7, and NKG2D regulate IFN-gamma secretion but not NKG2D-mediated cytotoxicity by human NK cells stimulated with suboptimal doses of IL12. J Immunol. 2007;179:3472-3479.

35. Sivori S, Falco M, Della Chiesa M, et al. CpG and double-stranded RNA trigger human NK cells by Toll-like receptors: induction of cytokine release and cytotoxicity against tumors and dendritic cells. Proc Nat Acad Sci USA. 2004;101:10116-10121.

36. Ziblat A, Domaica $\mathrm{Cl}$, Spallanzani RG, et al. IL-27 stimulates human NKcell effector functions and primes NK cells for IL-18 responsiveness. Eur J Immunol. 2015;45:192-202.

37. Ziblat A, Nunez SY, Raffo Iraolagoitia XL, et al. Interleukin (IL)-23 stimulates IFN-gamma secretion by CD56(bright) natural killer cells and enhances IL-18-driven dendritic cells activation. Front Immunol. 2017;8:1959.

38. Rabinovich BA, Li J, Shannon J, et al. Activated, but not resting, T cells can be recognized and killed by syngeneic NK cells. J Immunol. 2003;170:3572-3576.

39. Cerboni C, Zingoni A, Cippitelli M, et al. Antigen-activated human T lymphocytes express cell-surface NKG2D ligands via an ATM/ATRdependent mechanism and become susceptible to autologous NK- cell lysis. Blood. 2007;110:606-615.

40. Fousteri G, Dave Jhatakia A. Viral infections and autoimmune disease: roles of LCMV in delineating mechanisms of immune tolerance. Viruses 2019;11:885.

41. Abdel-Hakeem MS. Viruses teaching immunology: role of LCMV model and human viral infections in immunological discoveries. Viruses 2019;11:106.

42. Welsh RM, Brubaker JO, Vargas-Cortes M, et al. Natural killer (NK) cell response to virus infections in mice with severe combined immunodeficiency. The stimulation of NK cells and the NK cell-dependent control of virus infections occur independently of $\mathrm{T}$ and $\mathrm{B}$ cell function. J Exp Med. 1991;173:1053-1063.

43. Waggoner SN, Taniguchi RT, Mathew PA, et al. Absence of mouse 2B4 promotes NK cell-mediated killing of activated CD8+ T cells, leading to prolonged viral persistence and altered pathogenesis. J Clin Invest. 2010;120:1925-1938.

44. Lang PA, Lang KS, Xu HC, et al. Natural killer cell activation enhances immune pathology and promotes chronic infection by limiting CD8+ T-cell immunity. Proc Natl Acad Sci USA. 2012;109: 1210-1215.

45. Pallmer K, Barnstorf I, Baumann NS, et al. NK cells negatively regulate CD8 T cells via natural cytotoxicity receptor (NCR) 1 during LCMV infection. PLoS Pathog. 2019;15:e1007725.

46. Daniels KA, O'Donnell CL, Castonguay $C$, et al. Virus-induced natural killer cell lysis of T cell subsets. Virology. 2020;539:26-37.

47. Narni-Mancinelli E, Jaeger BN, Bernat C, et al. Tuning of natural killer cell reactivity by NKp46 and Helios calibrates T cell responses. Science. 2012;335:344-348.

48. Reddehase MJ, Lemmermann NAW. Mouse model of cytomegalovirus disease and immunotherapy in the immunocompromised host: predictions for medical translation that survived the "test of time." Viruses 2018;10:693.

49. Muntasell A, Vilches C, Angulo A, et al. Adaptive reconfiguration of the human NK-cell compartment in response to cytomegalovirus: a different perspective of the host-pathogen interaction. Eur J Immunol. 2013;43:1133-1141.

50. Waggoner SN, Cornberg M, Selin LK, et al. Natural killer cells act as rheostats modulating antiviral T cells. Nature. 2011;481: 394-398.

51. Hatfield SD, Daniels KA, O'Donnell CL, et al. Weak vaccinia virusinduced NK cell regulation of CD4 T cells is associated with reduced NK cell differentiation and cytolytic activity. Virology. 2018;519: 131-144.

52. Cook KD, Whitmire JK. The depletion of NK cells prevents T cell exhaustion to efficiently control disseminating virus infection. J Immunol. 2013;190:641-649.

53. Xu HC, Grusdat M, Pandyra AA, et al. Type I interferon protects antiviral CD8+ T cells from NK cell cytotoxicity. Immunity. 2014;40: 949-960.

54. Crouse J, Bedenikovic G, Wiesel M, et al. Type I interferons protect $\mathrm{T}$ cells against NK cell attack mediated by the activating receptor NCR1. Immunity. 2014;40:961-973.

55. Madera S, Rapp M, Firth MA, et al. Type I IFN promotes NK cell expansion during viral infection by protecting NK cells against fratricide. $J$ Exp Med. 2016;213:225-233.

56. Takao $\mathrm{S}$, Ishikawa $\mathrm{T}$, Yamashita $\mathrm{K}$, et al. The rapid induction of HLA-E is essential for the survival of antigen-activated naive CD4 T cells from attack by NK cells. J Immunol. 2010;185:6031-6040.

57. Schuster IS, Wikstrom ME, Brizard G, et al. TRAIL+ NK cells control $\mathrm{CD} 4+\mathrm{T}$ cell responses during chronic viral infection to limit autoimmunity. Immunity. 2014;41:646-656.

58. Peppa D, Gill US, Reynolds G, et al. Up-regulation of a death receptor renders antiviral T cells susceptible to NK cell-mediated deletion. J Exp Med. 2013;210:99-114.

59. Cardoso Alves L, Berger MD, Koutsandreas T, et al. Non-apoptotic TRAIL function modulates NK cell activity during viral infection. EMBO Rep. 2020;21:e48789.

60. Mehrotra PT, Donnelly RP, Wong S, et al. Production of IL-10 by human natural killer cells stimulated with IL-2 and/or IL-12. J Immunol. 1998;160:2637-2644.

61. Deniz G, Erten G, Kücüksezer UC, et al. Regulatory NK cells suppress antigen-specific T cell responses. J Immunol. 2008;180: 850-857.

62. Jinushi M, Takehara T, Tatsumi T, et al. Negative regulation of NK cell activities by inhibitory receptor CD94/NKG2A leads to altered NK cell-induced modulation of dendritic cell functions in chronic hepatitis C virus infection. J Immunol. 2004;173:6072-6081.

63. De Maria A, Fogli M, Mazza S, et al. Increased natural cytotoxicity receptor expression and relevant IL-10 production in NK cells 
from chronically infected viremic HCV patients. Eur J Immunol. 2007; 37:445-455

64. Li H, Zhai N, Wang Z, et al. Regulatory NK cells mediated between immunosuppressive monocytes and dysfunctional T cells in chronic HBV infection. Gut. 2018;67:2035-2044.

65. Rydyznski C, Daniels KA, Karmele EP, et al. Generation of cellular immune memory and B-cell immunity is impaired by natural killer cells. Nat Commun. 2015;6:6375.

66. Cook KD, Kline HC, Whitmire JK. NK cells inhibit humoral immunity by reducing the abundance of $C D 4+T$ follicular helper cells during a chronic virus infection. J Leukoc Biol. 2015;98:153-162.

67. Perona-Wright G, Mohrs K, Szaba FM, et al. Systemic but not local infections elicit immunosuppressive IL-10 production by natural killer cells. Cell Host Microbe. 2009;6:503-512.

68. Clark SE, Schmidt RL, McDermott DS, et al. A Batf3/NIrp3/IL-18 Axis Promotes Natural Killer Cell IL-10 Production during Listeria monocytogenes Infection. Cell Rep. 2018;23:2582-2594.

69. Maroof A, Beattie L, Zubairi S, et al. Posttranscriptional regulation of II10 gene expression allows natural killer cells to express immunoregulatory function. Immunity. 2008;29:295-305.

70. Clark SE, Filak HC, Guthrie BS, et al. Bacterial Manipulation of NK Cell Regulatory Activity Increases Susceptibility to Listeria monocytogenes Infection. PLoS Pathog. 2016;12:e1005708.

71. Lee S-H, Kim K-S, Fodil-Cornu N, et al. Activating receptors promote NK cell expansion for maintenance, IL-10 production, and CD8 T cell regulation during viral infection. J Exp Med. 2009;206:2235-2251.

72. Rydyznski CE, Cranert SA, Zhou JQ, et al. Affinity maturation is impaired by natural killer cell suppression of germinal centers. Cell Rep. 2018;24:3367-3373.e4.

73. Getts DR, Chastain EML, Terry RL, et al. Virus infection, antiviral immunity, and autoimmunity. Immunol Rev. 2013;255:197-209.

74. Fort MM, Leach MW, Rennick DM. A role for NK cells as regulators of CD4+ T cells in a transfer model of colitis. J Immunol. 1998;161: 3256-3261.

75. Chong WP, van Panhuys N, Chen J, et al. NK-DC crosstalk controls the autopathogenic Th17 response through an innate IFN- $\gamma$-IL-27 axis. J Exp Med. 2015;212:1739-1752.

76. Lu L, Ikizawa K, Hu D, et al. Regulation of activated CD4+ T cells by NK cells via the Qa-1-NKG2A inhibitory pathway. Immunity. 2007;26:593-604.

77. Leavenworth JW, Wang X, Wenander CS, et al. Mobilization of natural killer cells inhibits development of collagen-induced arthritis. Proc Natl Acad Sci USA. 2011;108:14584-14589.

78. Ehlers M, Papewalis C, Stenzel W, et al. Immunoregulatory natural killer cells suppress autoimmunity by down-regulating antigenspecific CD8+ T cells in mice. Endocrinology. 2012;153:4367-4379.

79. Morandi F, Horenstein AL, Chillemi A, et al. CD56brightCD16- NK cells produce adenosine through a CD38-mediated pathway and act as regulatory cells inhibiting autologous CD4+T cell proliferation. J Immunol. 2015;195:965-972.

80. Su HC, Nguyen KB, Salazar-Mather TP, et al. NK cell functions restrain T cell responses during viral infections. Eur J Immunol. 2001;31: 3048-3055.
81. Kim PS, Ahmed R. Features of responding T cells in cancer and chronic infection. Curr Opin Immunol. 2010;22:223-230.

82. Vitale M, Cantoni C, Pietra G, et al. Effect of tumor cells and tumor microenvironment on NK-cell function. Eur J Immunol. 2014;44: 1582-1592

83. Barber MA, Zhang T, Gagne BA, et al. NK cells negatively regulate antigen presentation and tumor-specific CTLs in a syngeneic lymphoma model. J Immunol. 2007;178:6140-6147.

84. Iyori M, Zhang T, Pantel H, et al. TRAIL/DR5 plays a critical role in NK cell-mediated negative regulation of dendritic cell cross-priming of $\mathrm{T}$ cells. J Immunol. 2011;187:3087-3095.

85. Iraolagoitia XLR, Spallanzani RG, Torres NI, et al. NK cells restrain spontaneous antitumor CD8+ T cell priming through PD-1/PD-L1 interactions with dendritic cells. J Immunol. 2016;197:953-961.

86. Terme M, Ullrich E, Aymeric L, et al. Cancer-induced immunosuppression: IL-18-elicited immunoablative NK cells. Cancer Res. 2012;72:2757-2767.

87. Dong W, Wu X, Ma S, et al. The mechanism of anti-PD-L1 antibody efficacy against PD-L1-negative tumors identifies NK cells expressing PDL1 as a cytolytic effector. Cancer Discov. 2019;9:1422-1437.

88. Soderquest $\mathrm{K}$, Walzer T, Zafirova B, et al. Cutting edge: CD8+ T cell priming in the absence of NK cells leads to enhanced memory responses. J Immunol. 2011;186:3304-3308.

89. Mailloux AW, Young MRI. NK-dependent increases in CCL22 secretion selectively recruits regulatory $T$ cells to the tumor microenvironment. J Immunol. 2009;182:2753-2765.

90. Crome SQ, Nguyen LT, Lopez-Verges S, et al. A distinct innate lymphoid cell population regulates tumor-associated T cells. Nat Med. 2017;23:368-375.

91. Picard E, Godet Y, Laheurte C, et al. Circulating NKp46+ natural killer cells have a potential regulatory property and predict distinct survival in non-small cell lung cancer. Oncoimmunology. 2019;8:e1527498.

92. Neo SY, Yang Y, Julien R, et al. CD73 immune checkpoint defines regulatory NK-cells within the tumor microenvironment. J Clin Invest. 2020;130:1185-1198.

93. Ostapchuk YO, Cetin EA, Perfilyeva YV, et al. Peripheral blood NK cells expressing HLA-G, IL-10 and TGF- $\beta$ in healthy donors and breast cancer patients. Cell Immunol. 2015;298:37-46.

94. Horenstein AL, Chillemi A, Zaccarello G, et al. A CD38/CD203a/CD73 ectoenzymatic pathway independent of CD39 drives a novel adenosinergic loop in human T lymphocytes. Oncoimmunology. 2013;2:e26246.

95. de Jonge K, Ebering A, Nassiri S, et al. Circulating CD56bright NK cells inversely correlate with survival of melanoma patients. Sci Rep. 2019;9:4487.

How to cite this article: Zwirner NW, Domaica $\mathrm{Cl}$, Fuertes MB. Regulatory functions of NK cells during infections and cancer. J Leukoc Biol. 2020;1-10. https://doi.org/10.1002/ JLB.3MR0820-685R 